\title{
Plasma exchange fails to improve outcomes for ANCA-associated vasculitis
}

Contrary to
expectations,
the use
of plasma
exchange did
not result
in a lower
incidence of
death or ESKD

The results of the PEXIVAS trial the largest ever clinical trial in the field of vasculitis - suggest that the addition of plasma exchange to induction immunosuppressive therapy does not lower the incidence of death or end-stage kidney disease (ESKD) in patients with anti-neutrophil cytoplasmic antibody (ANCA)-associated vasculitis (AAV). The trial data also revealed that a reduced-dose glucocorticoid regimen was non-inferior to standard dosing and resulted in fewer infection-related complications. Given that plasma exchange and glucocorticoids have both been widely used in the treatment of AAV, the findings, now published in The New England Journal of Medicine, could prove to be practice changing.

Previous trials had suggested that plasma exchange might reduce organ damage by rapidly removing ANCAs, but whether adding this treatment to immunosuppressive therapy would have a benefit with respect to survival or ESKD was unclear. Moreover, despite being an effective therapy for $\mathrm{AAV}$, the use of high-dose glucocorticoids is associated with dose-dependent adverse effects, and high-quality data regarding the rate at which glucocorticoid doses can be tapered in patients with AAV were lacking.

The PEXIVAS trial included 704 patients from 16 countries who had severe, active AAV and symptoms of kidney injury (defined by an estimated glomerular filtration rate of $<50 \mathrm{ml} / \mathrm{min}$ per $1.73 \mathrm{~m}^{2}$ of body-surface area) or diffuse pulmonary haemorrhage. The trial used a two-by-two factorial design that enabled the separate evaluation of initial treatment with plasma exchange versus no plasma exchange and of standarddose versus reduced-dose oral glucocorticoid therapy. All patients in the study received induction immunosuppressive therapy with either cyclophosphamide (intravenous or oral) or rituximab and were followed for up to 7 years for the primary composite outcome of death from any cause or ESKD.

Contrary to expectations, the use of plasma exchange did not result in a lower incidence of death or ESKD: the primary outcome occurred in $28 \%$ of patients who received plasma exchange compared with $31 \%$ of those who did not (HR 0.86; 95\% CI 0.65-1.13, $P=0.27)$. Secondary outcomes, including sustained remission, incidence of serious adverse events, incidence of infections and healthrelated quality of life outcome measures, also did not differ between the plasma exchange group and the control group.

For the comparison of the two glucocorticoid regimens, both groups received glucocorticoids for at least 52 weeks but the dose was tapered more rapidly in the reduced-dose group so that by 6 months the cumulative dose in this group was $<60 \%$ of that received in the standard-dose group. In the follow-up period, the primary outcome occurred in $28 \%$ of patients in the reduced-dose glucocorticoid group and $26 \%$ in the standard-dose group (absolute risk difference $2.3 \%$; $90 \%$ CI $-3.4 \%$ to $8.0 \%)$. The results confirmed the hypothesis that the reduced-dose regimen was non-inferior to the standard-dose regimen with respect to the risk of death or ESKD. Notably, patients in the reduced-dose group had a lower risk of serious infections in the first year of treatment (incidence rate ratio $0.69 ; 95 \% \mathrm{CI}$ $0.52-0.93$ ) but other secondary outcomes were similar across the two groups.

The findings suggest that the cost and inconvenience of plasma exchange is not warranted for the treatment of patients with $\mathrm{AAV}$, and that use of a reduced dose of glucocorticoids could reduce the risk of adverse events. The results of the PEXIVAS trial, regarding both the use of plasma exchange and glucocorticoid dosing, are expected to have an influence on the standard of care for patients with AAV.

Sarah Onuora

ORIGINAL ARTICLE Walsh, M. et al. Plasma exchange and glucocorticoids in severe ANCAassociated vasculitis. N. Engl. J. Med. 382, 622-631 (2020)

RELATED ARTICLES Derebail, V. K. \& Falk, R. J. ANCA-associated vasculitis - refining therapy with plasma exchange and glucocorticoids. N. Engl.J. Med. 382, 671-673 (2020)| McClure, M. et al. B cell therapy in ANCA-associated vasculitis: current and emerging treatment options. Nat. Rev. Rheumatol. 14, 580-591 (2018) 